\section{MINIMIZATION OF FUEL CONSUMPTION OF A SWARM OF SPACECRAFT THROUGH A GENETIC ALGORITHM APPROACH}

\author{
SHANE LECOMPTE, \\ DR. ANNALISA SCACCHIOLI (FACULTY ADVISOR)
}

\section{* ABSTRACT}

As humanity moves closer to forming realistic paths toward space exploration beyond that what we have already accomplished, multiple new challenges have presented themselves. Traditional large spacecraft prove to be unfeasible both logistically and economically for missions where a single problem can completely halt operations, especially given that higher reward missions are also of higher risk. A possible alternative to large craft is using a swarm of smaller craft made to accomplish the same goals while mitigating some of the drawbacks large craft face. Rockets, space shuttles, and satellites all prove to be too large to navigate areas of space dense with obstacles. Smaller craft on the scale of one meter in a large swarm would navigate these regions. Due to the decentralized nature of a swarm, any problems faced by one craft do not necessarily affect the others, allowing the swarm to stay operational despite some crafts becoming compromised. This feature means that a problem or miscalculation that could completely derail an entire mission in the context of a large spacecraft would not do the same to a swarm. In the context of exploring dense and/or extreme environments in space, many logistic and economic problems faced by large craft due to their size and centralized nature will not affect a swarm. With an accurate mathematical model of the swarm dynamics from Benet et al. ${ }^{[1]}$, a genetic algorithm's metaheuristic method is utilized ${ }^{[2]}$ to find optimal parameters that yield a minimal fuel consumption value for a given trajectory/mission objective. From this approach, the total fuel consumption was cut in half while retaining desirable characteristics of the trajectory such as collision avoidance and final formation constraints, giving us a similar course that accomplishes the same goal of transporting craft around objects and disturbances while also minimizing economic losses.

\section{$1 \quad$ INTRODUCTION}

Sending a large spacecraft to a destination far from Earth costs more money and has higher risk factors and implications of failure than the alternative presented in this paper. The materials fees, fuel cost for propelling the craft, and opportunity cost associated with large craft failure all heavily outweigh that of a swarm. Failure of any portion of the large craft can result in failure of the entire mission in a domino effect, while the swarm approach mitigates this. Sending a swarm through a field of densely packed asteroids or ice rocks in planetary rings involves a cooperative intelligence between individual agents that may not be as sophisticated on their own, but as a whole are comparable to any individual many orders of magnitude larger/smarter on its own. This idea is mimicked in nature with swarms of bees together to accomplish great feats of engineering comparable to humans despite their individual intelligence paling in comparison.

Despite the hackneyed image that space travel is an incredibly advanced and technical area for humankind, controlling a swarm of autonomous agents moving as a single unit was already solved by nature millions of years before humans ever existed. This trend can be seen in flocks of birds, schools of fish, and colonies of ants. They react to disturbances as a whole and can continue to survive even if individual swarm members are compromised. Following the biological inspiration for translating of this problem into a mathematical equation/model, a biologically inspired method of solving it seemed to be in order. The genetic algorithm is based on Darwin's theory of evolution. The idea of "survival of the 
fittest" as it is described in natural selection is utilized in this algorithm to only allow the most optimal members of each generation to survive to the next, thus giving rise to a criterion for convergence to a global optimum if this algorithm is run consecutively and recursively. These "members" are the parameters used in the differential equation model provided by Benet et al. ${ }^{[1]}$ and tweaked in this paper. Different values of certain parameters give rise to other solutions (trajectories). Thus, the genetic algorithm is utilized to find which parameters result in the most optimal trajectory.

In their paper, Conn et al. detail NASA's latest developments with autonomous spacecraft swarms. ${ }^{[3]}$ SODA, or Swarm Orbital Dynamics Advisor, is a computational framework for a control network that governs communications between spacecraft in a swarm. However, a fuel optimization approach is still yet to be fully explored in this context. Optimal path planning and fuel minimization approaches for UAVs (unmanned aerial vehicles) have been researched for terrestrial environments ${ }^{[4,5]}$, and in space in the context of transferring between multiple orbits ${ }^{[6]}$, but a combination of these approaches has yet to be extensively developed for space exploration in dynamically dense environments. In this paper, calculating the optimal trajectory of a swarm of spacecraft is investigated, minimizing the total fuel consumption in this specific context, thus finding both a logistic and economically viable alternative to that of traditional missions involving a singular large craft.

A specific area of space exploration, as mentioned earlier, in which the results of this work may be applicable is in the exploration of Saturn's rings. While we have been able to visit many moons of other planets in our solar system, we have only been able to visualize Saturn's rings from a distance due to the density and unpredictable size (meters to kilometers) of the ice rocks present in them. This environment provides an unacceptable risk for a multimillion-dollar spacecraft, so such missions have been avoided. A swarm of spacecraft with the same instrumental capabilities of a large vessel and collective intelligence equivalent to that of a larger computer system on a traditional craft that can morph around complex obstacles and dynamic obstacle patterns can provide a solution.

The total cost of the Cassini mission, a probe that orbited Saturn retrieving much of the data we have today about Saturn's rings, was about $\$ 3.26$ billion, with operations, fuel, and communications costing about $\$ 760$ million. ${ }^{[7]}$ This last figure is for the costs mostly associated with the Cassini mission only after it had entered Saturn's orbit and not the costs from the launch from Earth. With this being said, costs associated with launch and leaving Earth for both the traditional and swarm approach are the same considering the costs of operations in orbit are lessened by the swarm approach, not necessarily launch and manufacturing costs on Earth. The purpose of this paper is to highlight the economic viability of the swarm approach in the context of operations post-launch and during the actual mission. The swarm itself will most likely leave Earth on a larger craft and then be deployed from the mothercraft once the destination has been reached. Only then will the total cost of the entire project start to become much less than that of a traditional mission where a singular large craft leaves Earth and carries out the mission at the destination.

Not only will a swarm approach to this specific problem have the potential to cost much less, but it also has a much greater margin of error, resulting in less risk. There have been multiple studies showing both the economic and lower risk advantages of the swarm approach instead of the traditional large craft approach. ${ }^{[8,9]}$ Various work on the validity of swarm approaches to drone applications and general robotics applications has already been explored. ${ }^{[8,10,11]}$ In addition, NASA has considered projects involving "cubesats" that would accomplish similar goals using a swarm guidance framework within Earth's orbit, also including ideas for swarm missions to Saturn in particular to individual moons and the upper atmosphere, but never within the dense parts of the rings. ${ }^{[12]}$ In essence, a swarm approach not only can provide a method of getting more data about Saturn's rings to advance the science of planetary rings but can provide an economically viable and less risk-prone alternative to traditional mission outlines. 


\section{List of Relevant Terms}

ARTIFICIAL POTENTIAL FIELD - The assignment of values to every point in space that corresponds to the willingness of an object to move toward or away from that point in space while not being tied to any actual physical phenomena or interactions such as gravitation. A standard potential field encompasses these fundamental forces. ${ }^{[1]}$

BIFURCATION - The ability of a dynamic system to drastically change its behavior with the tuning of a single parameter. ${ }^{[1]}$

CONVERGENCE - When a system tends toward a certain steady value or state after a certain period of time.

LINE INTEGRAL - The integral of a function evaluated along a path in space. ${ }^{[14]}$

WORK - The amount of energy transfer associated with a force acting on an object, as calculated via the line integral of the force along a specified path in space. ${ }^{[14]}$

METAHEURISTIC - A computer search algorithm that is more sophisticated than simply checking all possible solutions in order to solve an optimization problem. ${ }^{[2]}$

Genetic Algorithm - A metaheuristic method of optimizing a function that is otherwise undefinable analytically or extremely difficult to do using methods of differential calculus. A global optimum is approximated numerically by mimicking Darwin's theory of evolution. With each successive generation, a more "fit" (lower fuel consumption) solution is created by taking the desirable characteristics of the previous generation and using them to pseudo-randomly generate the next. Each iteration (or generation) has "genes" associated with it that combine in a way with the possibility of mutation to produce the next generation. ${ }^{[2]}$

\section{METHODS \\ i. Mathematical Model}

In this paper, a swarm of twenty spacecraft is considered with dynamics modeled by a system of differential equations provided by Bennet et al. ${ }^{[1]}$ The model works for any number of craft, but twenty are used throughout this paper. The swarm is assumed to start stationed at a larger craft or satellite within space. The swarm will then be launched horizontally into space from this dock. This system of equations is derived from the basic principle of a particle's behavior in a potential field. In this model, at every point in three-dimensional space, a value for potential is defined, and in this case, the particles are individual spacecraft moving through this space. A potential field can be thought of as either a hill or a well where objects (particles) tend to either roll down the hill (move away from a higher potential) or roll into the well (move closer to a lower potential) wherein this analogy the potential is gravity. We can extend this same concept to a more abstract mathematical model by defining points in three-dimensional space that the spacecraft tend to move away from and others where the spacecraft tend to converge to. These artificial potentials depend on both the position of a craft in space as well as its relative positions to all others in the swarm. Suppose there are preset positions in space where low potentials occur in addition to high potentials being defined at the positions of each spacecraft and any obstacles. In that case, a mathematical scheme can be created where both external obstacle and inter-craft collision avoidance and convergence to desired final formations are well defined.

The following model (FIGURE 1) was based on the theory of bifurcating potential fields with a more rigorous mathematical derivation in the paper by Bennet et al..$^{[1]}$ They were able to derive a relation between the velocity of each craft in a conveniently defined (in the sense that it is artificially constructed with the final product in mind during its inception) potential field to get desired final formation and trajectory characteristics. Mathematically, the velocity is equal to the negative gradient of the potential field. The effects of these potential fields on the spacecraft 


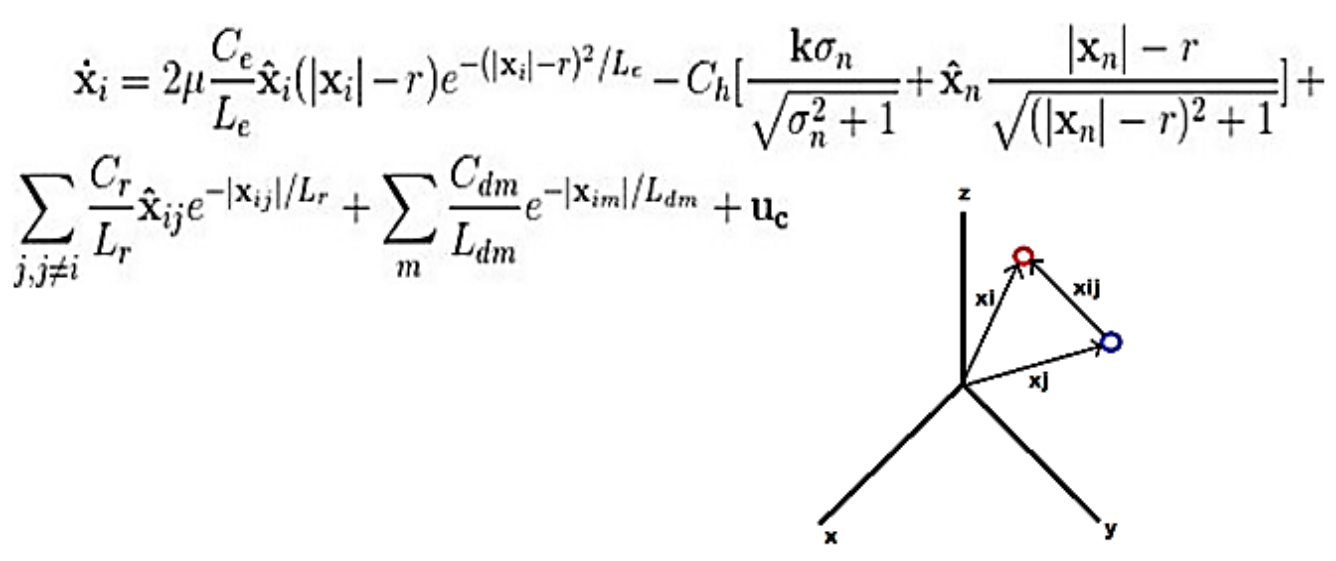

FIGURE 1: Mathematical model of swarm dynamics and reference system

only initiate the thrusters to force the spacecraft in a certain direction. In other words, these potentials are not real and are only defined within the computersensor framework of the swarm. The equation above is a modified version of the model from Bennet et al., ${ }^{[1]}$ taking this into consideration.

Above in FIGURE 1 the system of differential equations modeling the dynamics of the swarm is given. Without going into mathematical detail, these are a system of equations relating the velocities, positions, and relative positions of all swarm members to one another. The solution to this equation is the set of all the position functions of time of each member of the swarm, describing the path each craft follows through space. The diagram next to the model is the representation of the position vectors of two arbitrary crafts with respect to the origin and their relative position vector. This model contains four parts: the exponential potential, the hyperbolic potential, the inter-craft repulsive potential, and the modification, which we introduce as the craft-obstacle avoidance potential. The exponential and hyperbolic potentials control the final formation and trajectory, while the repulsive potential experienced by each craft governs obstacle and inter-craft avoidance. From this, a system of 20 first order, nonlinear, highly coupled, vector differential equations governing the motion of the swarm is obtained. Relevant variables and parameters are summarized in the following table (TABLE 1).

\section{ii. Formulation of Optimization Problem}

The main goal in this paper is to determine the values of certain parameters in the model that yield a trajectory that minimizes the total fuel consumption of the swarm. Fuel consumption in a craft is defined as being equal to the energy loss associated with a trajectory. Under the assumption that there is a direct proportion between fuel used and energy lost, the following calculations are all in joules per kilogram of fuel used. In other words, one kilogram of fuel used is equal to one joule of energy. This fact can be changed for any fuel/energy ratio for a real fuel with a simple multiplicative factor. In this case, energy loss is the work done on the craft by the thruster force along its trajectory. Since we neglect gravitation and any other forces acting on each craft, the only force acting on one craft is the force of its thrusters acting to either accelerate or decelerate it. Thus, the total work done by the thrusters on the craft will be equal to the amount of fuel used under these assumptions. The expression for work then that we are interested in is the following line integral evaluated along the path defined by the trajectory (FIGURE 2).

$$
\int \boldsymbol{F} \cdot d \mathbf{r}
$$

FIGURE 2: Work line integral 


\begin{tabular}{|c|c|}
\hline$a, b, c$ & PARAMETERS THAT ARE MEMBERS OF THE VECTOR K - control the shape of the final formation (circles, spheres, etc.) \\
\hline $\mathrm{C}_{\mathrm{h}}$ & $\begin{array}{l}\text { MAGNITUDE OF THE HYPERBOLIC POTENTIAL TERM - determines speed of convergence and works together with expo- } \\
\text { nential potential to determine general trajectory shape }\end{array}$ \\
\hline $\mathrm{C}_{\mathrm{e}}, \mathrm{L}_{\mathrm{e}}$ & $\begin{array}{l}\text { MAGNITUDE AND LENGTH SCALE OF EXPONENTIAL POTENTIAL TERM - work together with hyperbolic potential to deter- } \\
\text { mine general trajectory shapes and provide an upper bound on velocity }\end{array}$ \\
\hline $\mathrm{C}_{\mathrm{r}, \mathrm{Lr}}$ & $\begin{array}{l}\text { MAGNITUDE AND LENGTH SCALE OF INTER-CRAFT REPULSIVE POTENTIALS - determine how strong the repulsive force be- } \\
\text { tween crafts is. Constants of craft geometry and material }\end{array}$ \\
\hline $\mathrm{C}_{\mathrm{dm}, \mathrm{Ldm}}$ & $\begin{array}{l}\text { MAGNITUDE AND LENGTH SCALE OF CRAFT-OBSTACLE REPULSIVE POTENTIALS - determine how strong the repulsive force } \\
\text { between crafts and obstacles is. Constants of obstacle geometry }\end{array}$ \\
\hline$\mu$ & BIFURCATION PARAMETER - controls how many steady state formations swarm converges to \\
\hline $\mathrm{r}$ & $\begin{array}{l}\text { SCALAR - determines how spread out the swarm is as a whole by controlling locations of hyperbolic and expo- } \\
\text { tential potentials }\end{array}$ \\
\hline $\mathrm{Xi}_{\mathrm{i}}$ & POSITION VECTOR OF THE I'TH CRAFT \\
\hline $\mathrm{X}_{\mathrm{ij}}$ & RELATIVE POSITION VECTOR BETWEEN I'TH AND J'TH CRAFTS \\
\hline $\mathrm{u}_{\mathrm{x}}$ & AVERAGE VELOCITY WITH WHICH SWARM TRAVELS IN POSITIVE X-DIRECTION \\
\hline Xim & RELATIVE POSITION VECTOR BETWEEN I'TH CRAFT AND M'TH OBSTACLE \\
\hline
\end{tabular}

TABLE 1: Relevant parameters summary

Where $r$ is the displacement of the spacecraft and $\boldsymbol{F}$ is the net force experienced by the craft due to the thrusters along the path it follows to the end goal. We can then see that $d \boldsymbol{r} / d t$ is equal to the velocity of the craft traveling on this trajectory. We then arrive at $d \boldsymbol{r}=\boldsymbol{v} d t$. It is common to use in the field of dynamical systems to denote time derivatives with a small dot above the variable of interest ${ }^{[13]}$. The only forces exerted on the crafts being considered are the forces due to the thrusters. Gravitation from Saturn is neglected due to all particles and agents being within Saturn's orbital reference frame, where the force of gravity is already accounted for in defining this frame. Gravitation between spacecraft and ring particles is also neglected due to their extremely small masses that would result in small forces compared to the thruster forces. Since the variable " $x$ " denotes the position of a craft, " $x$ " with one dot denotes the velocity, while " $x$ " with two dots denotes acceleration. From this, and the fact that force is equal to mass times acceleration $(\boldsymbol{F}=m a)$ by
Newton's second law, we obtain the total work done by the thrusters in moving the craft along its trajectory as an integral from the starting time $(0)$ to the final time of the force vector. This pattern is expressed as the product of mass and the second time derivative (acceleration) of the i'th craft's displacement and $d \boldsymbol{r}$ being equal to $v d t$, which is equivalently expressed as the product of $d t$ and the first time derivative of the i'th craft's displacement. Finally, we sum over all craft to get the total energy lost (fuel consumed) of the swarm over the course of the trajectory (FIGURE 3).

$$
\sum_{i} \int_{0}^{t_{f}}\left|m \ddot{\mathbf{x}}_{i} \cdot \dot{\mathbf{x}}_{i}\right| d t
$$

FIGURE 3: Total energy expression of swarm along a trajectory 
Where $t_{f}$ is the final time during the simulation. The absolute value is necessary considering that if the thruster force is ever acting to decelerate a craft (it is acting opposite the direction of the craft's velocity), then the mathematical expression for work will yield a negative value, representing an energy gain in the system. Fuel is not generated in this instance, so the absolute value bars restrict this work to always represent an energy loss in the system.

The above expression is equal to the total energy lost during a swarm maneuver, representing the objective function we wish to minimize. Since this function is locked within a highly coupled system of differential equations and an integral that has no closed-form expression (an expression involving a finite amount of known algebraic operations and variables without derivatives and integrals), we must use a more advanced method of optimization which in this case is the genetic algorithm.

\section{iii. Genetic Algorithm}

In the genetic algorithm, a single member of a population is the set of parameters and its associated final fuel consumption value. We only consider the parameters $a, b, c, C_{e}, L_{e}, C_{h}$, and $r$ as the rest are either constants associated with the spacecraft or obstacles, which cannot be changed or are constants associated with a desired final formation type that we wish to keep constant.

The algorithm (refer to FIGURE 4) works by taking a set of random initial parameters, running them through a MATLAB Simulink ${ }^{[14]}$ simulation to obtain the fuel consumed in that trajectory, and then selecting the two sets of parameters that result in the lowest fuel consumed out of all of the initial sets. These two sets are chosen as the parents for the next generation. The parameters associated with these are then converted to a type of binary vector of ones and zeroes. The reason for this is because the next step

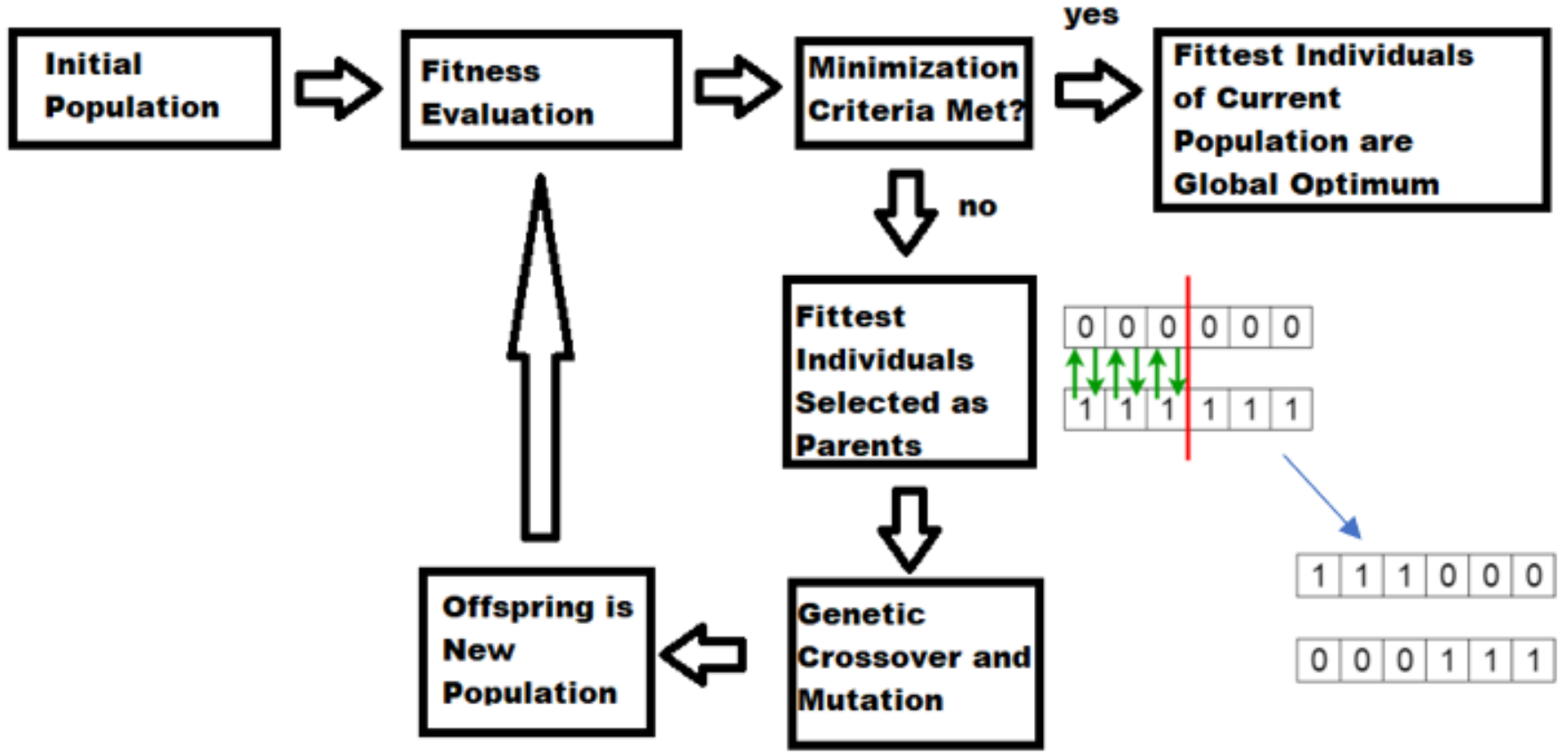

FIGURE 4: Genetic algorithm visualization ${ }^{[2]}$ 
is the exchanging of the "genetic material" of the two parents to produce offspring. The binary representations of each parent's parameter are lined up, and a random stopping point is then selected. This random stopping point is selected through a random number generator in the MATLAB code. Every value before the stopping point is swapped amongst the two parents, along with a random chance for one of the values to be inverted ( 1 to 0 or 0 to 1 ) at a low probability, representing random mutations. This process is continued a number of times until multiple "child" binary vectors are created, which are then converted back to their decimal representation. Together with this genetic crossover process and random mutations, there is a chance for every generation for a more optimal set of parameters to arise while not converging prematurely. After the conversion back to decimal form, the children are run through the same fitness test the previous population went through, and two new parents are selected while the other sets of parameters are scrapped. This process continues until a population converges to a global optimum where the fuel consumption values are all equally as fit as each other. Of course, other convergence criteria could be used (energy consumption below a certain value across the swarm, total time spent, etc.), but defining convergence in this way where every craft is equally optimal tends to prioritize the crafts expending the most fuel first. This process was implemented in MATLAB ${ }^{[14]}$ with multiple Simulink simulations running per generation to get the final fuel consumption value for each new set of parameters. Information and methods followed that were used to code this algorithm are presented in the article by Mallawaarachchi. ${ }^{[2]}$ A similar evolution-based optimization method was successfully used in path planning a single UAV in a terrestrial environment in the paper by Rathbun et al. ${ }^{[4]}$

\section{FUEL CONSUMPTION DEPENDS ON THE FORCE EXERTED ON A CRAFT BY THE THRUSTERS, WHICH IS PROPORTIONAL TO THE ACCELERATION OF THE CRAFT}

\section{NUMERICAL RESULTS}

In the following simulations, there is a swarm of twenty spacecraft initially arranged in a circle at the vertical launch pad and three static disturbances (ice rocks/asteroids) in space along the trajectory. In the context of Saturn's rings, this simulation represents a swarm being deployed from a mothercraft near Saturn into a region of Saturn's rings with ring particles acting as obstacles. It should be noted that this simulation and approach will work for any number of spacecraft and disturbances that may or may not be static. Still, for this work, a simpler scenario was chosen, primarily to demonstrate the efficacy of the chosen genetic algorithm in reducing fuel consumption. As described before, the fuel consumption depends on the force exerted on a craft by the thrusters, which is proportional to the acceleration of the craft. Whenever crafts are accelerating to form a final formation, avoid an obstacle, or avoid another craft, the thrusters must exert force on the craft to move it, thus spending fuel. Smoother trajectories generally use less fuel than trajectories where sharp turns and abrupt accelerations are required to avoid collisions. Within this preliminary model, collisions may happen and can be checked for but will not be physically accurate. The agents will move through obstacles or other craft upon collision. This simulation's main goal is to validate the genetic algorithm before making the mathematical model and environment too complicated. Future work on this project will involve the implementation of physically accurate collision physics and the event of a craft colliding and being compromised, leaving the swarm.

First, we start with an initial population of five sets of parameters, listed in the chart to the left of FIGURE 5. The trajectory and fuel consumption analysis for one craft is shown below in FIGURE 7. In FIGURE 5 we show the parameters used for this simulation as well as the swarm trajectory. FIGURE 6 presents an alternate view. As seen from FIGURE 5 and FIGURE 6, the swarm starts in an initial circular launch apparatus. It then moves through space approaching the final formation while avoiding the obstacles at locations denoted by large open circles and the other craft. The blue craft on the right provides an example of such aggressive avoidance behavior with an aggressive 

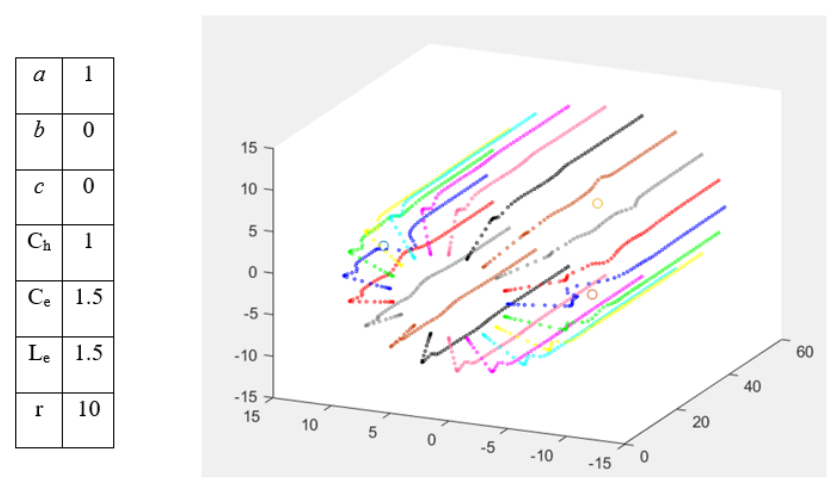

FIGURE 5: Parameters and associated trajectories of swarm traveling in $x$-direction. Different colored trajectories represent different spacecraft.

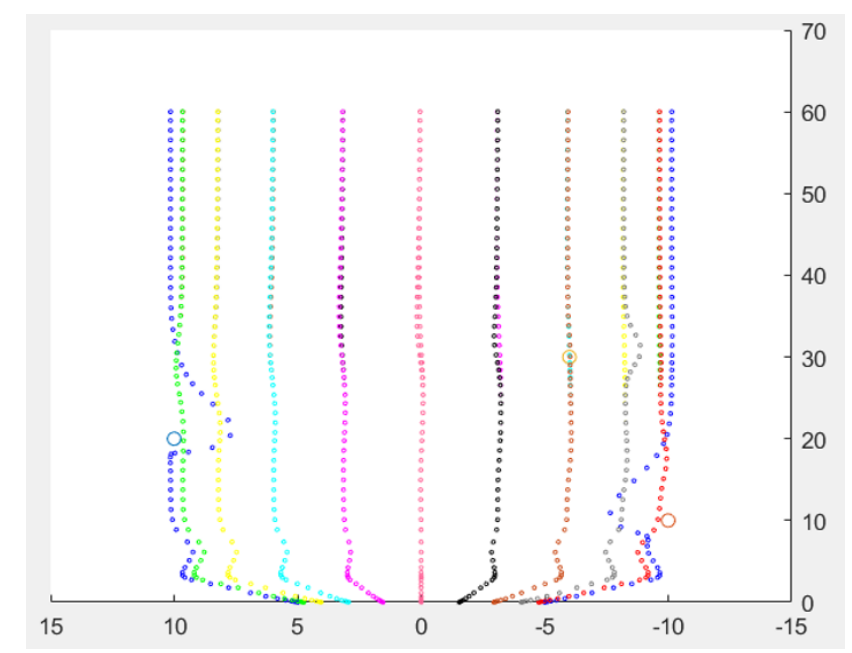

FIGURE 6: Top-Down View of Same Trajectory. Different colored trajectories represent different spacecraft.

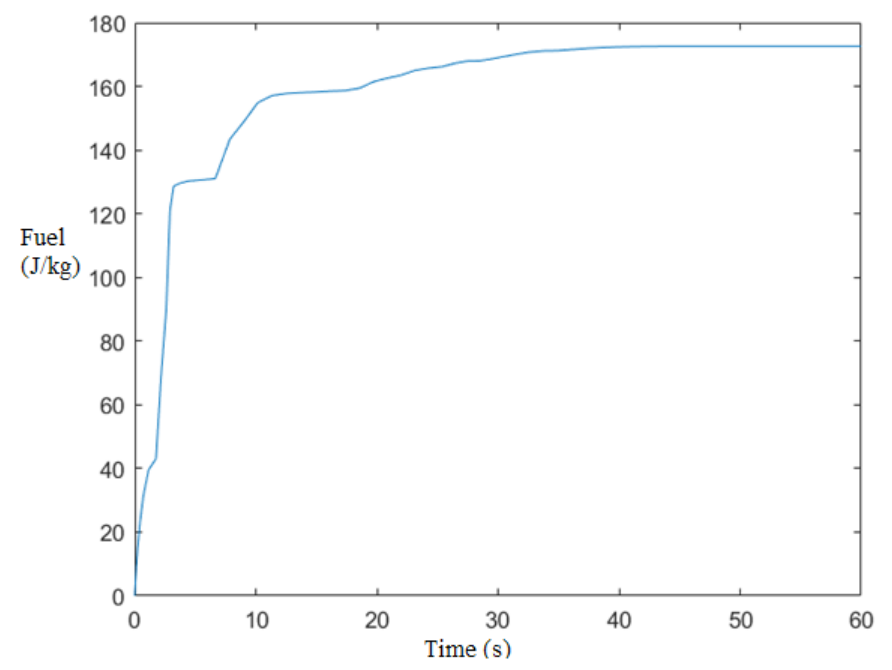

FIGURE 7: Fuel consumption ( $\mathrm{J} / \mathrm{kg}$ ) of one craft against time (s). maneuver at around 10 meters. The red trajectory next to it also appears to move out of the way of the blue one when it moves in the red trajectory's path to avoid the obstacle as well. This trend is better visible in FIGURE 5. As the blue craft dips inward toward the left, the red craft above it speeds up (more space in between consecutive dots represents faster movement) to avoid the blue craft. Although they are not necessarily on a collision course, they could be as these dots only represent the centers of spherical crafts, which could have varying sizes. The magnitudes of the inter-craft repulsive potentials responsible for this collision avoidance behavior would factor in the craft's radius when being chosen. In this case, the red and blue crafts became a bit too close to each other, so the avoidance mechanism governed by the potentials was initiated. In FIGURE 7 we show the fuel consumption against the time of this same blue craft described above.

A graph of a singular craft's fuel consumption is presented as it is still indicative of the swarm's consumption as a whole. This blue craft was the most responsible for increased fuel consumption in this trajectory due to its harsh avoidance maneuver. The fuel consumption spikes during periods of high acceleration and saturates once the swarm converges to the final formation as expected since the craft is no longer accelerating at that point. Also noteworthy is that fuel consumption spikes around when the blue trajectory aggressively avoids the obstacle (asteroid) bound for a head-on collision if it did not move out of the way. All of the fuel consumptions summed for each craft for this simulation was 3462.7 $\mathrm{J} / \mathrm{kg}$. This value is accurate within the model itself as it is a result of pure mathematical calculations without any data being taken.

After running this data set (set of the seven parameters being considered) and four others through the genetic algorithm, it converged to a possible optimal data set after 71 generations (or 355 individual simulations in Simulink). This process worked by taking the original population of five sets of the seven parameters being considered and running the Simulink simulation and corresponding total fuel usage for a swarm. With the five values of fuel consumption, the two smallest are considered and 
used as parents for the next generation. Using the technique described in the section on the genetic algorithm, five more sets of parameters (that have a higher chance of being optimal) are generated from the parents. One generation consists of the five simulations and calculations of total fuel consumption. So in total, the algorithm converged after 71 of these or 355 individual instances of numerically solving the system of equations and calculating integrals. The algorithm's optimal parameters output and their corresponding trajectories when run through the same simulation are described below in FIGURES 8 and 9.

One of the first things immediately made apparent is that the blue trajectories now no longer need to avoid the obstacle so aggressively, and the swarm as a whole being generally smoother as it converges to a final formation. With the use of the genetic algorithm, the swarm could navigate through the field of obstacles and arrive at the same destination while minimizing the fuel used to arrive there. In FIGURE 10 we display the fuel consumption versus time of the same craft as before, only now after the algorithm has run and the new parameters put in place.

The fuel consumption saturates in about half the time as the original simulation reaching a maximum value of a little less than $60 \mathrm{~J} / \mathrm{kg}$, which is much smaller than the original in FIGURE 7, being at about $170 \mathrm{~J} / \mathrm{kg}$. Another notable aspect of this new trajectory is that the fuel consumption curve in FIGURE $10 \mathrm{in}$ creases much more smoothly than that of the unoptimized swarm, which has many abrupt spikes. In the context of a real-world scenario, the same mission could be completed for half the fuel cost while also putting less strain on the thrusters.

\section{CONCLUSIONS}

Although these are preliminary results, they show that the genetic algorithm approach can be successfully optimize the fuel consumption of a swarm trajectory of spacecraft defined by this complex nonlinear model. The solution to this problem provides a method of exploring and gaining new information on the rings of Saturn that otherwise would not be possible. Future work will include making the model more realistic and transitioning the
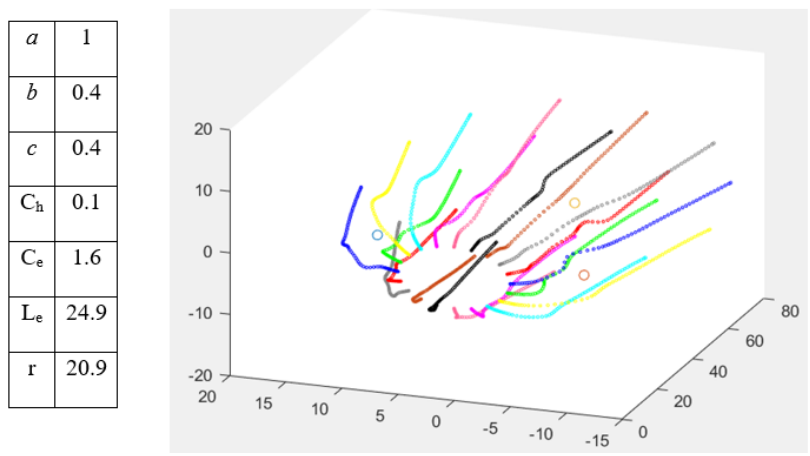

FIGURE 8: Parameters and associated trajectories of swarm after genetic algorithm. Different colored trajectories represent different spacecraft.

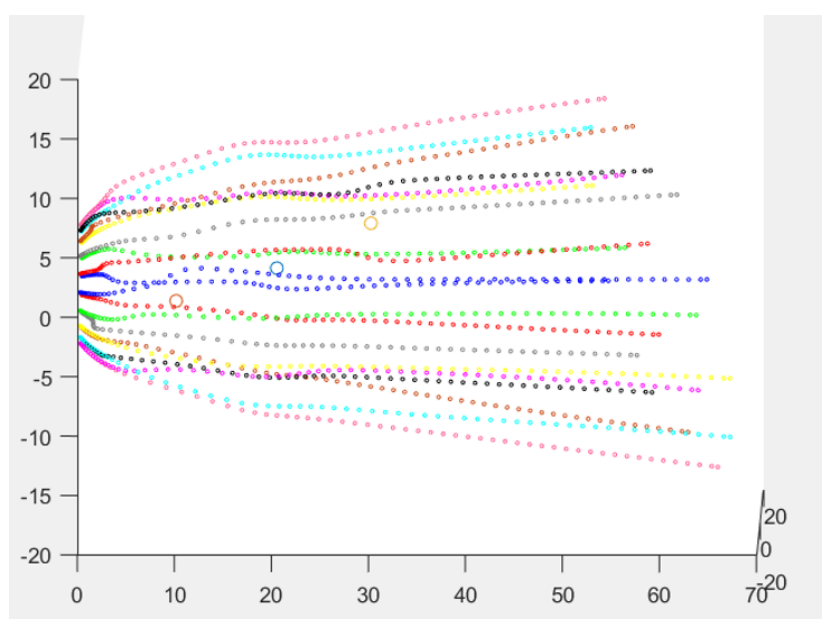

FIGURE 9: Side view of same trajectory. Different colored trajectories represent different spacecraft.

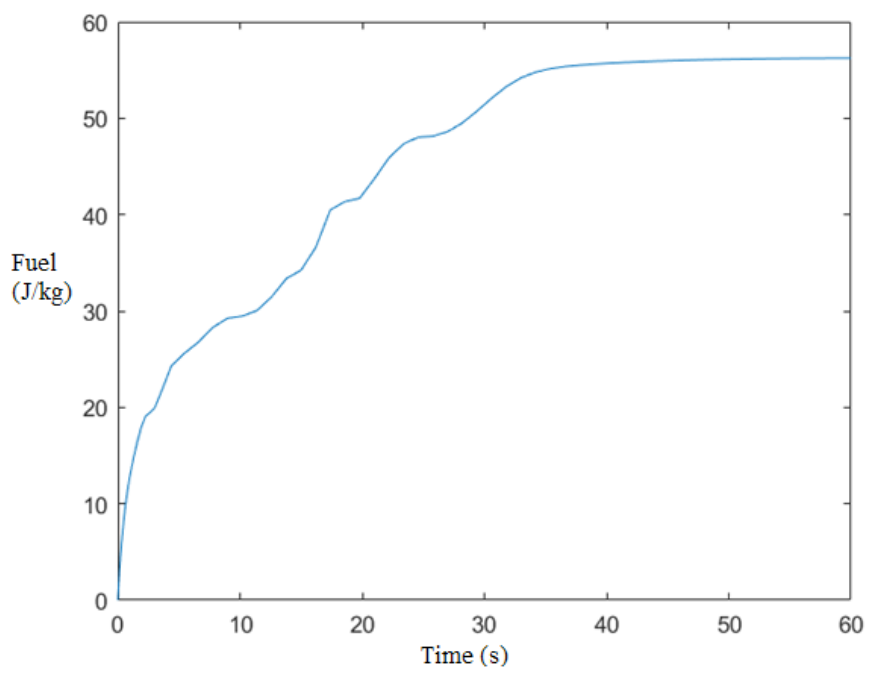

FIGURE 10: Fuel consumption ( $\mathrm{J} / \mathrm{kg}$ ) of one craft against time (s) after genetic algorithm. 
deterministic problem to a stochastic one; denser obstacle distributions, and obstacles that are moving, will also be considered in future simulations more akin to the real-world density of ice rocks in the rings of Saturn. Applying the genetic algorithm method to the same spacecraft swarm system described by more accurate stochastic models can lead to future innovative developments in this field. New methods of optimization will also be investigated

\section{ACKNOWLEDGEMENTS}

I would first like to thank Dr. Annalisa Scacchioli for guiding me through this process and opening up this wonderful opportunity to learn as well as create something meaningful. Without her, this project would not have come to fruition. I would also like to thank the NASA New Jersey Space Grant Consortium for funding and facilitating this research. Also, I would like to thank Dr. Jingang Yi as well as Dr. Enver Koray Akdogan for providing insight into the inner workings of the mathematical model used in this paper as well as possible avenues to best utilize it for this project.

\section{REFERENCES}

[1] Bennet, Derek J., Mclnnes, C.R., Suzuki, M., and Uchiyama K., "Autonomous Three-Dimensional Formation Flight for a Swarm of Unmanned Aerial Vehicles," Journal of Guidance, Control, and Dynamics, Vol. 34, No. 6, November-December 2011, 1899-1908.

[2] Mallawaarachchi, Vijini, "Introduction to Genetic Algorithms - Including Example Code," Medium Towards Data Science, 7 July 2017.

[3] Conn, T., Plice L., Dono Perez, A., and Ho, M., "Operating Small Sat Swarms as a Single Entity: Introducing SODA," NASA Ames Research Center / Mission Design Division, 31 $1^{\text {st }}$ Annual AIAA/USU Conference on Small Satellites, 2017.

[4] Rathbun, David, Kragelund Sean, and Pongpunwattana, Anawat, "An Evolution Based Path Planning Algorithm for Autonomous Motion of a UAV Through Uncertain Environments," University of Washington, Seattle, WA, 2002.

[5] Zhao, Yiyuan J. and Celia Qi, Ying, "Minimum Fuel Powered Dynamic Soaring of Unmanned Aerial Vehicles Utilizing Wind Gradients," University of Minneapolis, MN, 2004.

[6] Morgan, Daniel, Chong, Soon-Jo, and Hadaegh, Fred Y., "Model Predictive Control of Swarms of Spacecraft Using Sequential Convex Programming," Journal of Guidance, Control, and Dynamics, Vol. 37, No 6, November-December 2014, 1725-1740.
[7] "Cassini-Solstice Mission FAQs," Jet Propulsion Laboratory. Retrieved January 24, NASA, 2014.

[8] Blocher, A., "Alternative Mission Concepts for the Exploration of Outer Planets," California Polytech State University, Master's Thesis 2017.

[9] Hadaegh, F. Y., Chung, S., Manohara, H. M., "On Development of 100-Gram Class Spacecraft for Swarm Applications," IEEE Systems Journal, Vol. 10, No. 2, Pages 673-684, June 2016, 2016.

[10] Hinchley, M. G., Rash, J. L., Truszkowski, W. F.,"Autonomous and Autonomic Swarms," Information Systems Division, NASA Goddard Space Flight Center, Technical Report, 2006.

[11] Nallapu, R., Thangavelautham, J., "Design of Spacecraft Swarm Flybys for Planetary Moon Exploration," Structures, Structural Design and Materials Conference, Technical Report, 2019.

[12] Blocher, A., Atkinson, D., Freeman, T., "Saturn Swarm Study Small Probe and CubeSat Architectures to accompany New Frontiers missions at Saturn" Low Cost Planetary Mission Conference, Presentation, 2017.

[13] MATLAB Simulink, math and computer algebra system, coding interface, and simulation environment, Mathworks, 2019.

[14] Hibbeler, Russel C., Engineering Mechanics: Dynamics, $14^{\text {th }}$ Edition, 2016. 